\title{
A SINGULAR CRITICAL POTENTIAL FOR THE SCHRÖDINGER OPERATOR
}

\author{
THOMAS DUYCKAERTS
}

\begin{abstract}
We construct a potential $V$ on $\mathbb{R}^{d}$, smooth away from one pole, and a sequence of quasimodes for the operator $-\Delta+V$, which concentrate on this pole. No smoothing effect, Strichartz estimates nor dispersive inequalities hold for the corresponding Schrödinger equation.
\end{abstract}

\section{INTRODUCTION}

Consider a Schrödinger operator:

$$
P=-\Delta+V, \quad \Delta=\sum_{j=1}^{d} \frac{\partial^{2}}{\partial x_{j}^{2}},
$$

on $\mathbb{R}^{d}, d \geq 1$, where $V$ is a real potential, small at infinity, and the initial value problem for the Schrödinger equation:

$$
\left\{\begin{array}{c}
i \partial_{t} U-P U=0 \\
U_{i t=0}=U_{0} \in L^{2}\left(\mathbb{R}^{d}\right) .
\end{array}\right.
$$

For sufficiently smooth potentials $V$, the equation (2) implies a local smoothing effect:

$$
\|\chi U\|_{L^{2}(] 0, T\left[, H^{1 / 2}\left(\mathbb{R}^{d}\right)\right)} \leq C\left\|U_{0}\right\|_{L^{2}\left(\mathbb{R}^{d}\right)}, \chi \in C_{0}^{\infty}\left(\mathbb{R}^{d}\right),
$$

where $T$ may be finite, and in some cases infinite, and $C$ may depend on $V, \chi$ and $T$. This well-known estimate (see [2, 6] and references therein) is essential in the study of (2) and related non-linear equations. In this paper, we investigate the minimal regularity to be assumed on $V$ such that (31) is still true. In 6], A. Ruiz and L. Vega consider the equation (2) as a perturbation of the free Schrödinger equation to show an inequality which implies (3). The following potentials fall within this scope:

- $V \in L^{p}, p \geq d / 2$.

- Potentials belonging to some Morey-Campanato spaces, (which are larger than $L^{d / 2}$ ) with a smallness condition in these spaces (see [6] for details). For example, potentials with inversesquare singularities:

$$
V=\sum_{j=1 . . N} \frac{a_{j}}{\left|x-p_{j}\right|^{2}}+V_{0}, V_{0} \in L^{p}, p \geq d / 2,
$$

where the $\left|a_{j}\right|$ 's are small enough.

Let us mention that potentials in the article above may be time-dependent but we shall not develop this point here. In [5], (see also [3]), the author studies potentials of type (4) with regular $V_{0}$, assuming only the following positivity properties on the $a_{j}$ 's:

$$
a_{j}+(d / 2-1)^{2}>0, \quad j=1 . . N,
$$

and shows non-trapping type inequality on the truncated resolvent:

$$
\forall \lambda \in \mathbb{C}, \operatorname{Re} \lambda^{2} \geq C, \operatorname{Im} \lambda^{2} \neq 0, \quad\left\|\chi\left(P-\lambda^{2}\right)^{-1} \chi\right\|_{L^{2} \rightarrow L^{2}} \leq \frac{C}{|\lambda|}, \quad \chi \in C_{0}^{\infty}\left(\mathbb{R}^{d}\right),
$$

One may deduce from (5) estimates of type (3) (cf [2]). 
The preceding works do not study potentials $V \in L^{p}, p<d / 2$, and potentials of type (4) but with poles of higher order. In this paper, we show that the space $L^{d / 2}$ and the inverse-square singularities are critical, by constructing an unipolar potential whose pole is of the order of $(\log r)^{2} / r^{2}, r=|x|$, near 0 , and such that (3), (5) and some other usual estimates on solutions of Schrödinger equations do not hold.

Theorem 1. Let $d \geq 1, N \geq 0$ be integers. There exist:

- a radial, positive potential $V$ on $\mathbb{R}^{d}$, which has compact support and such that:

$$
\begin{gathered}
V \in C^{\infty}\left(\mathbb{R}^{d} \backslash\{0\}\right) \\
\frac{|\log r|^{2}}{C r^{2}} \leq V(r) \leq \frac{C|\log r|^{2}}{r^{2}}, r \leq r_{0},
\end{gathered}
$$

- an increasing sequence $\left(\lambda_{n}\right)_{n \geq n_{0}}$ of positive real numbers, diverging to $+\infty$,

- a sequence of radial $C^{\infty}$ functions $\left(u_{n}\right)_{n \geq n_{0}}$, whose support is of the following form:

$$
\left\{c_{1} \frac{\log \lambda_{n}}{\lambda_{n}} \leq r \leq c_{2} \frac{\log \lambda_{n}}{\lambda_{n}}\right\}
$$

such that:

$$
\begin{gathered}
(-\Delta+V) u_{n}-\lambda_{n}^{2} u_{n}=f_{n} \\
\left\|u_{n}\right\|_{L^{1}}=1 \\
\forall j \in \mathbb{N},\left\|\frac{d^{j}}{d r^{j}} f_{n}\right\|_{L^{\infty}}=O\left(\lambda_{n}^{j-N}\right), n \rightarrow+\infty .
\end{gathered}
$$

Corollary 1. Let $N$ be any integer greater than $1, P=-\Delta+V$, where $V$ is the potential of the preceding theorem, and $\chi$ a function in $C_{0}^{\infty}\left(\mathbb{R}^{d}\right)$ which does not vanish in 0 . Then:

- (lack of local Strichartz estimates)

(11) $\forall q, q_{0} \in[1,+\infty], q>q_{0}, \forall T>0, \forall C>0, \exists U_{0} \in C_{0}^{\infty},\|\chi U(t)\|_{L^{1}(] 0, T\left[, L^{q}\left(\mathbb{R}^{d}\right)\right)}>C\left\|U_{0}\right\|_{L^{q_{0}\left(\mathbb{R}^{d}\right)}}$;

- (lack of local smoothing effect)

- (lack of local dispersion)

- (lack of Strichartz estimates with loss of derivative)

$$
\begin{aligned}
\forall q \in[1,+\infty], \forall \sigma \in[0,1], & \frac{1}{2}-\frac{1}{q}>\frac{\sigma}{d}, \\
& \forall T>0, \forall C>0, \exists U_{0} \in C_{0}^{\infty},\|\chi U(t)\|_{L^{1}(] 0, T\left[L^{q}\left(\mathbb{R}^{d}\right)\right)}>C\left\|U_{0}\right\|_{D\left(P^{\sigma / 2}\right)} .
\end{aligned}
$$

In the preceding statements, we wrote $U(t)$ the solution of the equation (2) with initial value $U_{0}$ and $q^{\prime}$ the conjugate exponent of $q$ which is defined by: $1 / q+1 / q^{\prime}=1$.

Remarks.

- If $d>2$, the hypothesis (6) and (7) implie:

$$
V \in \bigcap_{p<d / 2} L^{p}
$$

- Of course, the sequence $\left(f_{n}\right)_{n \geq n_{0}}$ invalidates (5) when $N \geq 2$.

- It will be clear in the proof of the theorem than one may construct quasi-modes of infinite order (i.e. such that (10) holds with $O\left(\lambda_{n}^{-\infty}\right)$ instead of $O\left(\lambda_{n}^{j-N}\right)$ ) by taking a singularity just a bit stronger:

$$
\frac{|\log r|^{2+\varepsilon}}{C r^{2}} \leq V(r) \leq \frac{C|\log r|^{2+\varepsilon}}{r^{2}}, \varepsilon>0 .
$$

With a still stronger singularity, one may force $f_{n}$ to be exponentially decreasing in $-\lambda_{n}$. 
- Classical Strichartz estimates:

$$
\|u\|_{L^{p}(] 0, T\left[L^{q}\left(\mathbb{R}^{d}\right)\right)} \leq C\left\|u_{0}\right\|_{L^{2}\left(\mathbb{R}^{d}\right)}, p>2, \frac{2}{p}+\frac{d}{q}=\frac{d}{2},
$$

are invalidated by (11), with the exception of the case $q=2, p=+\infty$ which is always true by the $L^{2}$ conservation law. Likewise, (14) shows that Strichartz estimates with loss of derivative (see [2]) of the form:

$$
\|u\|_{L^{p}(] 0, T\left[L^{q}\left(\mathbb{R}^{d}\right)\right)} \leq C\left\|u_{0}\right\|_{D\left(P^{\sigma / 2}\right)}, \frac{1}{2}-\frac{1}{q}>\frac{\sigma}{d}, \sigma \in[0,1],
$$

are false. In this inequality, the limit case $\sigma / d=1 / 2-1 / q$ is an immediate consequence of the Sobolev inclusion

$$
L^{q}\left(\mathbb{R}^{d}\right) \subset H^{\sigma}\left(\mathbb{R}^{d}\right) \subset D\left(P^{\sigma / 2}\right),
$$

and of the conservation of the norm $D\left(P^{\sigma / 2}\right)$ of any solution of (2). Notice that this last inclusion implies that (14) is still true in usual Sobolev spaces, i.e when $D\left(P^{\sigma / 2}\right)$ is replaced by $H^{\sigma}\left(\mathbb{R}^{n}\right)$.

- The potential $V$ and quasi-modes $u_{n}$ being of compact support, as close to 0 as desired, the preceding counter-example is still valid in any regular domain, for a Laplace operator with Dirichlet or Neumann Dirichlet boundary condition.

- Examples of linear Schrödinger equations which do not admit the classical dispersive inequality or Strichartz estimates are given in 11 and 4. In these two cases, the particular behaviour of the equation arises from the metric which defines the Laplace operator. Let us mention that the idea to use quasi-modes in relation with Strichartz inequalities is owed to M. Zworski (see [8]).

Finally, we would like to point out that for the potential $V$ introduced in the theorem, one may define the operator $P$ without ambiguity. The operator $-\Delta+V$ has a natural meaning on $C_{0}^{\infty}\left(\mathbb{R}^{d} \backslash\{0\}\right)$, and is essentially self-adjoint on this space, under a positivity asumption implied by (7). We call $P$ its unique self-adjoint extension, which is of course positive. We refer to Reed and Simon [7, Th X.30] for any precision.

The author would like to thank Nicolas Burq, its advisor and Clotilde Fermanian Kammerer for their precious help.

Section 2 of the paper is devoted to the proof of the corollary, and section 3 to that of the theorem.

\section{Proof of the Corollary.}

According to Hölder's inequality, for any function $v$ on $\mathbb{R}^{d}$, with a finite volume support:

$$
\|v\|_{L^{q_{0}}} \leq B^{1 / q_{0}-1 / q}\|v\|_{L^{q}}, \quad q>q_{0},
$$

where $B$ is the volume of the support. Let $U_{n}(t)=e^{-i \lambda_{n}^{2} t} u_{n}$. Then:

$$
\begin{gathered}
i \partial_{t} U_{n}-P U_{n}=-e^{-i \lambda_{n}^{2} t} f_{n}, \quad U_{n\lceil t=0}=u_{n} \\
U_{n}(t)=e^{-i t P} u_{n}+i e^{-i \lambda_{n}^{2} t} \int_{0}^{t} e^{i(s-t) P} f_{n} d s .
\end{gathered}
$$

Assume that (11) is false. According to (16):

$$
\int_{0}^{T}\left\|\chi U_{n}(t)\right\|_{L^{q}\left(\mathbb{R}^{d}\right)} d t \leq \int_{0}^{T}\left\|\chi e^{-i t P} u_{n}\right\|_{L^{q}\left(\mathbb{R}^{d}\right)} d t+\int_{0}^{T} \int_{0}^{t}\left\|\chi e^{i(s-t) P} f_{n}\right\|_{L^{q}\left(\mathbb{R}^{d}\right)} d s d t .
$$

Since the support of $u_{n}$ concentrates in 0 , and $\chi$ does not vanish in 0 , the left term of this inequality is, for $n$ large enough, greater than:

$$
\frac{1}{C} \int_{0}^{T}\left\|u_{n}\right\|_{L^{q}\left(\mathbb{R}^{d}\right)} d t \geq \frac{1}{C}\left(\frac{\log \lambda_{n}}{\lambda_{n}}\right)^{d\left(1 / q-1 / q_{0}\right)}\left\|u_{n}\right\|_{L^{q_{0}\left(\mathbb{R}^{d}\right)}} .
$$


Using the negation of (11), the right term is dominated by:

$$
\left\|u_{n}\right\|_{L^{q_{0}\left(\mathbb{R}^{d}\right)}}+\left\|f_{n}\right\|_{L^{q_{0}\left(\mathbb{R}^{d}\right)}} .
$$

Hence we obtain, using (10):

$$
\left(\frac{\lambda_{n}}{\log \lambda_{n}}\right)^{d\left(1 / q_{0}-1 / q\right)}\left\|u_{n}\right\|_{L^{q_{0}}}=O\left(\left\|u_{n}\right\|_{L^{q_{0}}}+\lambda_{n}^{-N}\right),
$$

which leads to the announced contradiction since $N \geq 1$ and $q>q_{0}$, the norm of $u_{n}$ in $L^{q_{0}}$ being greater than 1 by (9).

By Sobolev inequalities, (11) implies (12), taking in (11) $q_{0}=2$ and $q>2$ close enough to 2. So (12) holds.

Let us assume that (13) is not true. Then we get, by (16):

$$
\begin{aligned}
\left\|\chi u_{n}(T)\right\|_{L^{q}\left(\mathbb{R}^{d}\right)} & \leq C\left\|u_{n}\right\|_{L^{q^{\prime}\left(\mathbb{R}^{d}\right)}}+\int_{0}^{T}\left\|\chi e^{i(s-T)} f_{n}\right\|_{L^{1}\left(\mathbb{R}^{d}\right)} d s \\
& \leq C\left(\left\|u_{n}\right\|_{L^{q^{\prime}\left(\mathbb{R}^{d}\right)}}+\left\|f_{n}\right\|_{L^{2}\left(\mathbb{R}^{d}\right)}\right) .
\end{aligned}
$$

To obtain the second inequality we have bounded, up to a multiplicative constant, the $L^{q^{\prime}}$ norm on the support of $\chi$ by the $L^{2}$ norm. The contradiction is again a simple consequence of (15) and the fact that $q^{\prime}<q$.

We shall now prove (14). Otherwise, we would have by (16):

$$
\int_{0}^{T}\left\|u_{n}(t)\right\|_{L^{q}\left(\mathbb{R}^{d}\right)} d t \leq C\left\|u_{n}\right\|_{D\left(P^{\sigma / 2}\right)}+\int_{0}^{T} \int_{0}^{t}\left\|e^{i(s-t) P} f_{n}\right\|_{D\left(P^{\sigma / 2}\right)} d s d t .
$$

Of course, $e^{i(s-t) P}$ maps isometrically the domain of $P^{\sigma / 2}$ onto itself. We have:

$$
\left\|f_{n}\right\|_{D\left(P^{1 / 2}\right)}^{2}=\int\left|\nabla f_{n}(x)\right|^{2} d x+\int(1+V(x))\left|f_{n}(x)\right|^{2} d x
$$

On the support of $f_{n}$, as on that of $u_{n},|x| \geq \frac{\log \lambda_{n}}{C \lambda_{n}}$. Using the superior bound of $V$ in (17), we get, on the support of $f_{n}$ :

$$
|V(r)| \leq C \frac{\lambda_{n}^{2}}{\left(\log \lambda_{n}\right)^{2}}\left(\log \log \lambda_{n}-\log \lambda_{n}\right)^{2} \leq C \lambda_{n}^{2} .
$$

It follows using (10) and $N \geq 2$ that:

$$
\left\|f_{n}\right\|_{D\left(P^{1 / 2}\right)} \leq C\left(\left\|\nabla f_{n}\right\|_{L^{2}}+\lambda_{n}\left\|f_{n}\right\|_{L^{2}}\right) \leq C \lambda_{n}^{-1} .
$$

Furthermore, the equation $P u_{n}-\lambda_{n}^{2} u_{n}=f_{n}$ implies, using once again the bounds (9) and (10) on the norms of $u_{n}$ and $f_{n}$ :

$$
\begin{aligned}
\left\|u_{n}\right\|_{D\left(P^{1 / 2}\right)}^{2} & \leq\left\|f_{n}\right\|_{L^{2}}\left\|u_{n}\right\|_{L^{2}}+\lambda_{n}^{2}\left\|u_{n}\right\|_{L^{2}}^{2} \\
& \leq C \lambda_{n}^{2}\left\|u_{n}\right\|_{L^{2}}^{2} .
\end{aligned}
$$

By interpolation on the norms of the left hand side, and since $0 \leq \sigma \leq 1$, we get:

$$
\left\|u_{n}\right\|_{D\left(P^{\sigma / 2}\right)} \leq C \lambda_{n}^{\sigma}\left\|u_{n}\right\|_{L^{2}}
$$

According to (18), (19) and the inequality (17):

$$
\left\|u_{n}\right\|_{L^{q}} \leq C \lambda_{n}^{\sigma}\left\|u_{n}\right\|_{L^{2}}+o(1) .
$$

Hence, with (15):

$$
\left(\frac{\lambda_{n}}{\log \lambda_{n}}\right)^{d / 2-d / q}\left\|u_{n}\right\|_{L^{2}} \leq C \lambda_{n}^{\sigma}\left\|u_{n}\right\|_{L^{2}}+o(1),
$$

which is absurd since $\frac{d}{2}-\frac{d}{q}>\sigma$. 


\section{DÉmonstration DU ThÉORÈme.}

Denote by $r$ the euclidian norm of $x$ and by ' the radial derivative $\frac{d}{d r}$. We would like to find radial functions $V, f_{n}, u_{n}$ such that:

$$
f_{n}(r)=-u_{n}^{\prime \prime}(r)-\frac{d-1}{r} u_{n}^{\prime}(r)+V(r) u_{n}(r)-\lambda_{n}^{2} u_{n}(r),
$$

with $f_{n}$ small and $\lambda_{n}$ diverging to infinity. We shall first change functions to get rid of the first-order derivative in this equation. Set:

$$
u_{n}=r^{-\frac{d-1}{2}} v_{n}, \quad f_{n}=r^{-\frac{d-1}{2}} g_{n}, \quad W=V+\frac{d^{2}-4 d+3}{4 r^{2}} .
$$

Thus (20) becomes:

$$
g_{n}(r)=-v_{n}^{\prime \prime}+W v_{n}-\lambda_{n}^{2} v_{n}
$$

Let:

$$
y(s)=e^{-\sqrt{s^{2}+1}}, b(s)=-\frac{1}{\left(s^{2}+1\right)^{3 / 2}}+\frac{s^{2}}{s^{2}+1},
$$

which are $C^{\infty}$ solutions of the equation on $\mathbb{R}$ :

$$
\begin{gathered}
-y^{\prime \prime}(s)+b(s) y(s)=0 \\
y(s)>0, \forall j \in \mathbb{N}, \quad\left|y^{(j)}(s)\right| \leq C_{j} e^{-|s|} \\
|b(s)| \leq 1 .
\end{gathered}
$$

We shall write:

$$
y_{\omega, a}(r)=y(\omega(r-a)), \quad b_{\omega, a}(r)=\omega^{2} b(\omega(r-a)),
$$

where $a$ and $\omega$ are two real parameters. We have:

$$
-y_{\omega, a}^{\prime \prime}+b_{\omega, a} y_{\omega, a}=0
$$

Let $q(\lambda)$ be a strictly increasing positive function defined in a neighbourhood of $+\infty$ such that:

$$
\begin{gathered}
\lim _{\lambda \rightarrow+\infty} q(\lambda)=+\infty \\
\lim _{\lambda \rightarrow+\infty} \frac{q(\lambda)}{\lambda}=0 .
\end{gathered}
$$

Let $\left(\lambda_{n}\right)_{n \geq n_{0}}$ be the sequence defined by the equations:

$$
10^{n}=q\left(\lambda_{n}\right),
$$

so that $\lambda_{n}$ diverges faster to $+\infty$ than $10^{n}$.

Choose a cutoff function $\chi: \chi \in C_{0}^{\infty}(]-1,1[), \chi=1$ on $\left[-\frac{1}{2}, \frac{1}{2}\right]$.

Set:

$$
\begin{gathered}
\psi_{n}(r)=\chi\left(10^{n} r\right)-\chi\left(10^{n+1} r\right), \\
\chi_{n}(r)=\chi\left(10^{n+1}\left(r-\frac{3}{10^{n+1}}\right)\right),
\end{gathered}
$$




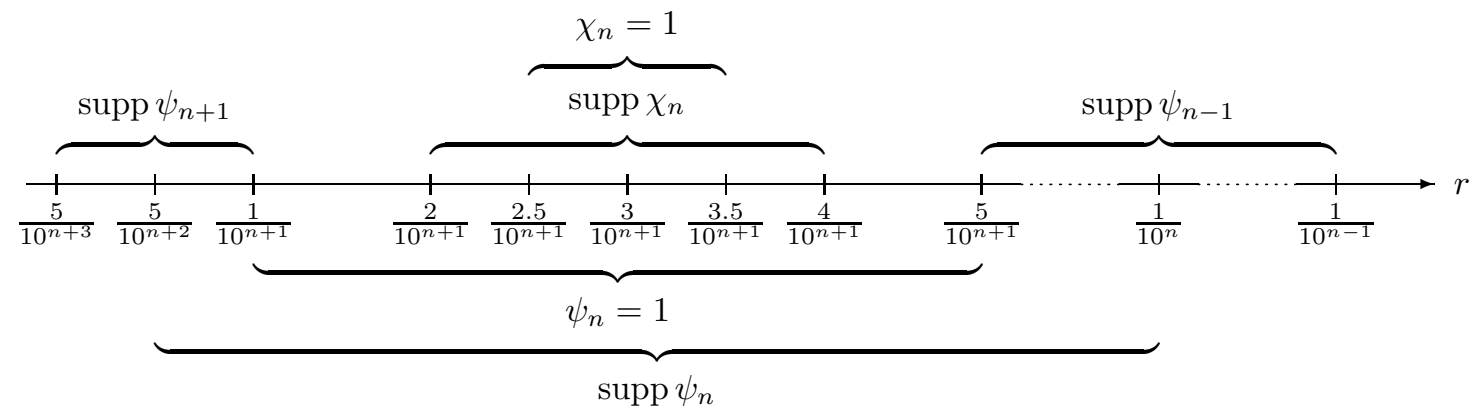

Figure 1. Cutoff functions near $3 / 10^{n+1}$

The $\psi_{n}$ 's form a partition of unity near 0 . The support of each $\chi_{n}$ is included in that of $\psi_{n}$, away from those of the $\psi_{j}$ 's, $j \neq n$ :

$$
\begin{gathered}
\operatorname{supp} \psi_{n} \subset\left[\frac{5}{10^{n+2}}, \frac{1}{10^{n}}\right] \\
\frac{1}{10^{n+1}} \leq r \leq \frac{5}{10^{n+1}} \Rightarrow \psi_{n}(r)=1 \\
\sum_{n \geq n_{0}} \psi_{n}(r)=\chi\left(10^{n_{0}} r\right) \\
\operatorname{supp} \chi_{n} \subset\left[\frac{2}{10^{n+1}}, \frac{4}{10^{n+1}}\right] \subset\left\{\psi_{n}=1\right\} \\
\frac{25}{10^{n+2}} \leq r \leq \frac{35}{10^{n+2}} \Rightarrow \chi_{n}(r)=1 .
\end{gathered}
$$

We shall denote by $y_{n}$ and $b_{n}$ the following functions:

$$
\begin{gathered}
y_{n}(r)=y_{\frac{\lambda_{n}}{2}, \frac{3}{10^{n+1}}}(r)=y\left(\frac{\lambda_{n}}{2}\left(r-\frac{3}{10^{n+1}}\right)\right) \\
b_{n}(r)=b_{\frac{\lambda_{n}}{2}, \frac{3}{10^{n+1}}}(r)=\frac{\lambda_{n}^{2}}{4} b\left(\frac{\lambda_{n}}{2}\left(r-\frac{3}{10^{n+1}}\right)\right) .
\end{gathered}
$$

Each of the function $-b_{n}$ may be seen as a potential well which concentrates (by equation (2211) the energy of $y_{n}$ near $3 / 10^{n+1}$. We shall construct $W$ so that the equation $g_{n}(r)=0$ is exactly $-v_{n}^{\prime \prime}+b_{n} v_{n}=0$ on a small interval (including the support of $v_{n}$ ) around the point $3 / 10^{n+1}$. The size of this interval will be of the same order as $10^{-n}$, smaller than the equation parameter $\omega=\lambda_{n} / 2$. We shall choose $v_{n}$ as a cutoff of $y_{n}$. The exponential decay of $y$, combined with the difference of order between these two scales will induced small enough error terms for (9) and (10) to hold.

Set (see the figure):

$$
\begin{gathered}
W(r)=\sum_{n \geq n_{0}} \psi_{n}(r)\left(b_{n}(r)+\lambda_{n}^{2}\right) \\
v_{n}(r)=\alpha_{n} y_{n}(r) \chi_{n}(r),
\end{gathered}
$$

where the $\alpha_{n}$ 's are strictly positive constants yet to be determined. The functions $g_{n}, u_{n}, f_{n}$ and the potential $V$ are thus defined by (201) and (21). 


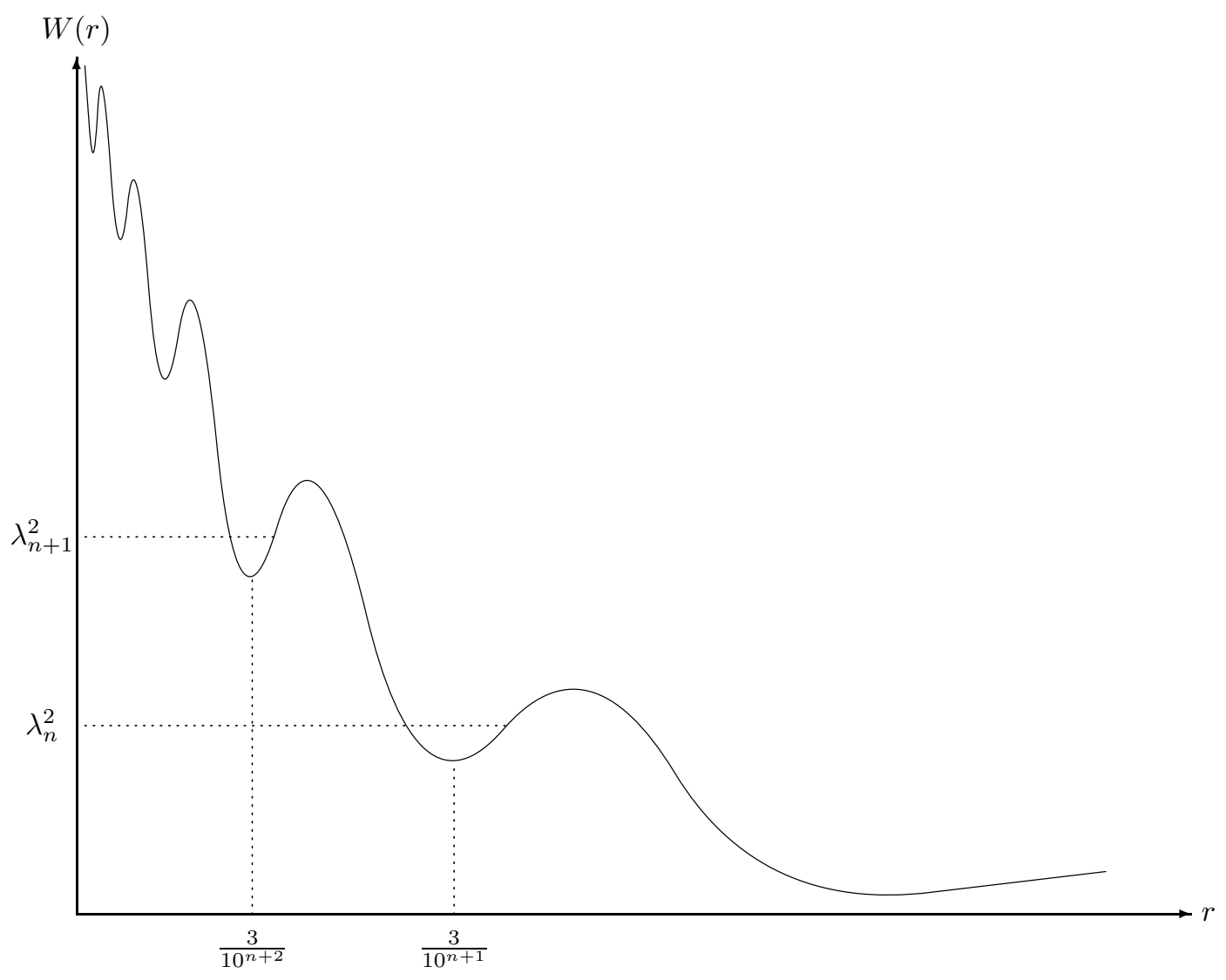

Figure 2. The potential W

Lemma 1. The following inequalities hold:

$$
\begin{gathered}
\forall j \in \mathbb{N}, \exists C>0,\left\|\frac{d^{j} f_{n}}{d r^{j}}\right\|_{L^{\infty}\left(\mathbb{R}^{d}\right)} \leq C \alpha_{n} \lambda_{n}^{1+j}\left(q\left(\lambda_{n}\right)\right)^{\frac{d+1}{2}} e^{-\frac{\lambda_{n}}{20 q\left(\lambda_{n}\right)}} \\
\left\|u_{n}\right\|_{L^{1}\left(\mathbb{R}^{d}\right)} \geq \frac{\alpha_{n}}{C} \lambda_{n}^{-1}\left(q\left(\lambda_{n}\right)\right)^{-\frac{d-1}{2}} .
\end{gathered}
$$

Proof. According to (28), on the support of $\chi_{n}, W$ is equal to $b_{n}+\lambda_{n}^{2}$. Hence:

$$
g_{n}(r)=-v_{n}^{\prime \prime}(r)+b_{n}(r) v_{n}(r) .
$$

Thus, using (221):

$$
f_{n}(r)=r^{-\frac{d-1}{2}} g_{n}(r)=r^{-\frac{d-1}{2}} \alpha_{n}\left(y_{n}(r) \chi_{n}^{\prime \prime}(r)+2 y_{n}^{\prime}(r) \chi_{n}^{\prime}(r)\right) .
$$

The derivative of $f_{n}$ of order $j$ is then of the form:

$$
\frac{d^{j} f_{n}}{d r^{j}}=\sum_{j_{1}+j_{2}+j_{3}=j+1} \beta_{j_{1}, j_{2}, j_{3}} \frac{d^{j_{1}}}{d r^{j_{1}}} y_{n} \frac{d^{j_{2}}}{d r^{j_{2}}} \chi_{n}^{\prime} \frac{d^{j_{3}}}{d r^{j_{3}}} r^{-\frac{d-1}{2}} .
$$

On the support of $\chi_{n}^{\prime}$ :

$$
\left|r-\frac{3}{10^{n+1}}\right|>\frac{1}{10^{n+1}}=\frac{1}{10 q\left(\lambda_{n}\right)} .
$$


So according to the bounds (23) of $y$ and its derivatives:

$$
\begin{aligned}
\left|y_{n}^{\left(j_{1}\right)}(r)\right| & \leq C \lambda_{n}^{\left(j_{1}\right)} e^{-\frac{\lambda_{n}}{2}\left|r-\frac{3}{10^{n}+1}\right|} \\
& \leq \lambda_{n}^{j_{1}} e^{-\frac{\lambda_{n}}{20 q\left(\lambda_{n}\right)}}
\end{aligned}
$$

Furthermore,

$$
\begin{gathered}
\left|\chi_{n}^{\left(j_{2}+1\right)}\right| \leq C\left(q\left(\lambda_{n}\right)\right)^{j_{2}+1} \leq C \lambda_{n}^{j_{2}} q\left(\lambda_{n}\right) \\
\left|\left(\frac{d}{d r}\right)^{j_{3}}\left(r^{-\frac{d-1}{2}}\right)\right| \leq C\left(q\left(\lambda_{n}\right)\right)^{\frac{d-1}{2}+j_{3}} \leq C \lambda_{n}^{j_{3}}\left(q\left(\lambda_{n}\right)\right)^{\frac{d-1}{2}},
\end{gathered}
$$

for on the support of $\chi_{n}, r \geq \frac{1}{10^{n+1}}$. These three inequalities, together with (37), implies (34).

We shall now prove (35). By the definition of $y_{n}$ :

$$
\frac{\lambda_{n}}{2}\left|r-\frac{3}{10^{n}}\right| \leq \frac{1}{2} \Rightarrow y_{n}(r) \geq m=\sup _{|s| \leq \frac{1}{2}}|y(s)| .
$$

Furthermore, if $r$ is as above, and $n$ big enough, then $\chi_{n}(r)=1$ and so:

$$
u_{n}(r)=r^{-\frac{d-1}{2}} v_{n}(r)=r^{-\frac{d-1}{2}} \alpha_{n} y_{n}(r)
$$

Hence:

$$
\begin{aligned}
\left\|u_{n}\right\|_{L^{1}} & \geq m \alpha_{n} \int_{\lambda_{n}\left|r-\frac{3}{10^{n}}\right| \leq 1} r^{-\frac{d-1}{2}} r^{d-1} d r \\
& \geq \frac{\alpha_{n}}{C}\left(10^{-n}\right)^{\frac{d-1}{2}} \lambda_{n}^{-1} .
\end{aligned}
$$

Choose $M>1$ and set:

$$
q(\lambda)=\frac{\lambda}{M \log \lambda} .
$$

The positive function $q$ is strictly increasing for big $\lambda$ 's and satisfies (25) and (26). In addition, lemma 1 implies, bounding from above $q\left(\lambda_{n}\right)$ by $\lambda_{n}$ :

$$
\begin{gathered}
\left\|\frac{d^{j} f_{n}}{d r^{j}}\right\|_{L^{\infty}} \leq C \alpha_{n} \lambda_{n^{\frac{d+3}{2}}+j-\frac{M}{20}} \\
\left\|u_{n}\right\|_{L^{1}} \geq \frac{\alpha_{n}}{C} \lambda_{n}^{\frac{d+1}{2}}
\end{gathered}
$$

with new constants $C$, which may depend on $M$. So the conditions (9) and (10) of the theorem are satisfied if the constants $M$ and $\alpha_{n}$ are well chosen. The support of $u_{n}$ is that of $\chi_{n}$, which is of the desired form:

$$
\left\{c_{1} \frac{\log \lambda_{n}}{\lambda_{n}} \leq r \leq c_{2} \frac{\log \lambda_{n}}{\lambda_{n}}\right\},
$$

if the support of $\chi$ is taken to be a segment.

The assertion (7) on the potential remains to be checked. We have the following approximation of the inverse function of $q$ :

Lemma 2. Let $q$ be defined by (38). Then:

$$
q(\lambda) \log (q(\lambda)) \sim \frac{\lambda}{M}, \lambda \rightarrow+\infty .
$$


Proof.

$$
\begin{aligned}
q(\lambda) \log q(\lambda) & =q(\lambda) \log \left(\frac{\lambda}{M \log \lambda}\right) \\
& =q(\lambda)(\log \lambda-\log \log \lambda-\log M) \\
& \sim q(\lambda) \log \lambda,
\end{aligned}
$$

when $\lambda$ goes to infinity.

On the support of $\psi_{n}, q\left(\lambda_{n}\right)=10^{n}$ and so:

$$
\begin{gathered}
\frac{1}{20 r} \leq q\left(\lambda_{n}\right) \leq \frac{1}{r} \\
\frac{1}{20 r}|\log r-\log 20| \leq q\left(\lambda_{n}\right) \log \left(q\left(\lambda_{n}\right)\right) \leq \frac{1}{r}|\log r| .
\end{gathered}
$$

(We used that $s \mapsto s \log s$ is an increasing function for $s>e^{-1}$ ). Using lemma 2 and taking $r$ close enough to 0 we get:

$$
C^{-1} \frac{1}{r}|\log r| \leq \lambda_{n} \leq C \frac{1}{r}|\log r| .
$$

Thus, by the definition (32) of $W,\left|b_{n}\right|$ being bounded from above by $\lambda_{n}^{2} / 4$ :

$$
C^{-1} \frac{|\log r|^{2}}{r^{2}} \sum_{n \geq n_{0}} \psi_{n}(r) \leq W(r) \leq C \frac{|\log r|^{2}}{r^{2}} \sum_{n \geq n_{0}} \psi_{n}(r)
$$

which implies, with (27), the inequality (7) on the potential $W$. Consequently $V$ satisfies the same inequality.

Remark. To get quasi-modes of infinite order, we would have taken $q(\lambda)=\frac{\lambda}{(\log \lambda)^{1+\varepsilon}}$ and suitably modified lemma 2

\section{REFERENCES}

[1] V. Banica. Dispersion and Strichartz inequalities for Schrödinger equations with singular coefficients. A paraître au SIAM Journal of Mathematical Analysis.

[2] N. Burq, P. Gérard, N. Tzvetkov. On nonlinear Schrödinger equations in exterior domains. Preprint.

[3] N. Burq, F. Planchon, J.G. Stalker, A.Shadi Tahvildar-Zadel. Strichartz estimates for the wave and Schrödinger equations with the inverse-square potential. Preprint.

[4] C. Castro, E. Zuazua. Concentration and lack of observability of waves in highly heterogeneous media. Arch. Ration. Mech. Anal. 164 (2002), No 1, 39-72.

[5] T. Duyckaerts. Inégalités de résolvante pour des potentiels multipolaires. Travail en cours.

[6] A. Ruiz, L. Vega. Local regularity of solutions to wave with time-dependent potentials. Duke Math. J. 76 (1994) No 3 p. 913-940.

[7] M. Reed, B. Simon. Methods of modern mathematical physics. 2, Fourier analysis, self-adjointness. San Diego CA London Sydney: Academic Press, 1975.

[8] H. F. Smith, C.D. Sogge, Global Strichartz estimates for nontrapping perturbations of the Laplacian. Comm. Partial Differential Equations 25 (2000), No 11-12, 2171-2183. Arch. Ration. Mech. Anal. 164 (2002), No 1, 39-72.

Université de Paris-Sud, BÂtiment 430, 91405 Orsay Cedex

E-mail address: Thomas.Duyckaerts@math.u-psud.fr 


\title{
POTENTIEL SINGULIER CRITIQUE POUR L'OPÉRATEUR DE SCHRÖDINGER
}

\author{
THOMAS DUYCKAERTS
}

\begin{abstract}
RÉSumÉ. On construit un potentiel $V$ sur $\mathbb{R}^{d}$, régulier en dehors d'un pôle, et une suite de quasi-modes pour l'opérateur $-\Delta+V$, qui se concentre sur ce pôle. Les solutions de l'équation de Schrödinger associée ne vérifient pas d'effet régularisant local, ni d'inégalités de Strichartz ou de dispersion.
\end{abstract}

\section{INTRODUCTION}

On considère un opérateur de la forme :

$$
P=-\Delta+V, \quad \Delta=\sum_{j=1}^{d} \frac{\partial^{2}}{\partial x_{j}^{2}},
$$

sur $\mathbb{R}^{d}, d \geq 1$, où $V$ est un potentiel réel, petit à l'infini, et l'équation de Schrödinger :

$$
\left\{\begin{array}{c}
i \partial_{t} U-P U=0 \\
U_{\gg t=0}=U_{0} \in L^{2}\left(\mathbb{R}^{d}\right) .
\end{array}\right.
$$

Lorsque $V$ est suffisamment régulier, l'équation (2) entraîne un effet régularisant local :

$$
\|\chi u\|_{L^{2}(] 0, T\left[, H^{1 / 2}\left(\mathbb{R}^{d}\right)\right)} \leq C\left\|u_{0}\right\|_{L^{2}\left(\mathbb{R}^{d}\right)}, \chi \in C_{0}^{\infty}\left(\mathbb{R}^{d}\right),
$$

où selon les cas $T$ est fini ou infini, et $C$ peut dépendre de $V$, $\chi$ et $T$. Cette estimation classique ( $\operatorname{cf}[2],[6]$ et les références proposées dans ces deux articles) est fondamentale dans l'étude de (2) et des équations non-linéaires associées. On s'intéresse à la régularité minimale de $V$ pour que (3) soit encore vérifiée. Dans 6], A. Ruiz et L. Vega considèrent l'équation (2) comme une perturbation de l'équation de Schrödinger libre pour démontrer (3) (en fait une inégalité plus forte). Les potentiels suivants rentrent dans ce cadre :

- $V \in L^{p}, p \geq d / 2$.

- Des potentiels dans des classes de Morey-Campanato, plus larges que $L^{d / 2}$, vérifiant une condition de petitesse dans ces espaces (cf [6] pour les détails). Par exemple, des potentiels admettant des singularités ponctuelles de la forme

$$
V=\sum_{j=1 . . N} \frac{a_{j}}{\left|x-p_{j}\right|^{2}}+V_{0}, V_{0} \in L^{p}, p \geq d / 2
$$

où les $\left|a_{j}\right|$ sont petits.

Les potentiels étudiés dans cet article peuvent aussi dépendre du temps mais nous ne développerons pas cet aspect ici. Dans [5], (voir aussi [3]), l'auteur étudie des potentiels de type [4), (avec $V_{0}$ régulier) en faisant simplement l'hypothèse de positivité sur les $a_{j}$ :

$$
a_{j}+(d / 2-1)^{2}>0,
$$

et démontre des inégalités sur la résolvante tronquée :

$$
\forall \lambda \in \mathbb{C}, \operatorname{Re} \lambda^{2} \geq C, \operatorname{Im} \lambda^{2} \neq 0, \quad\left\|\chi\left(P-\lambda^{2}\right)^{-1} \chi\right\|_{L^{2} \rightarrow L^{2}} \leq \frac{C}{|\lambda|}, \quad \chi \in C_{0}^{\infty}\left(\mathbb{R}^{d}\right),
$$

On peut notamment en déduire des estimations de type (3) (cf [2]). 
Les potentiels $V \in L^{p}, p<d / 2$, et ceux de la forme de (4), mais avec des pôles d'ordre supérieur ne sont pas traités dans les travaux précités. On montre ici le caractère critique de l'espace $L^{d / 2}$ et des singularités en $1 / r^{2}, r=|x|$, en construisant un potentiel unipolaire $V$ dont le pôle est de l'ordre de $|\log r|^{2} / r^{2}$ près de 0 , tel que $P$ ne vérifie ni (3) ni (5).

Théorème 1. Soient $d \geq 1, N \geq 0$ des entiers. Il existe :

- un potentiel $V$ sur $\mathbb{R}^{d}$, positif, radial, à support compact tel que :

$$
\begin{gathered}
V \in C^{\infty}\left(\mathbb{R}^{d} \backslash\{0\}\right) \\
\frac{|\log r|^{2}}{C r^{2}} \leq V(r) \leq \frac{C|\log r|^{2}}{r^{2}}, r \leq r_{0} .
\end{gathered}
$$

- une suite $\left(\lambda_{n}\right)_{n \geq n_{0}}$ croissante de réels strictement positifs, de limite $+\infty$;

- une suite de fonctions $\left(u_{n}\right)_{n \geq n_{0}}$, radiales, $C^{\infty}$, dont le support est de la forme :

$$
\left\{c_{1} \frac{\log \lambda_{n}}{\lambda_{n}} \leq r \leq c_{2} \frac{\log \lambda_{n}}{\lambda_{n}}\right\}
$$

tels que:

$$
\begin{gathered}
(-\Delta+V) u_{n}-\lambda_{n}^{2} u_{n}=f_{n} \\
\left\|u_{n}\right\|_{L^{1}}=1 \\
\forall j \in \mathbb{N},\left\|\frac{d^{j}}{d r^{j}} f_{n}\right\|_{L^{\infty}}=O\left(\lambda_{n}^{j-N}\right), n \rightarrow+\infty .
\end{gathered}
$$

Corollaire 1. Soient $P=-\Delta+V$, où $V$ est le potentiel introduit dans le théorème pour un $N \geq 2$, et $\chi \in C_{0}^{\infty}\left(\mathbb{R}^{d}\right)$ non nulle en 0 . Alors :

- (absence d'inégalités de Strichartz locales)

(11) $\forall q, q_{0} \in[1,+\infty], q>q_{0}, \forall T>0, \forall C>0, \exists U_{0} \in C_{0}^{\infty},\|\chi U(t)\|_{L^{1}(] 0, T\left[, L^{q}\left(\mathbb{R}^{d}\right)\right)}>C\left\|U_{0}\right\|_{L^{q_{0}}\left(\mathbb{R}^{d}\right)} ;$

- (absence d'effet régularisant local)

$$
\forall \sigma>0 \forall T>0, \forall C>0, \exists U_{0} \in C_{0}^{\infty},\|\chi U(t)\|_{L^{1}(] 0, T\left[, H^{\sigma}\left(\mathbb{R}^{d}\right)\right)}>C|| U_{0} \|_{L^{2}\left(\mathbb{R}^{d}\right)} ;
$$

- (absence de dispersion locale)

$$
\forall q \in] 1,+\infty], \forall T>0, \forall C>0, \exists U_{0} \in C_{0}^{\infty},\|\chi U(T)\|_{L^{q}\left(\mathbb{R}^{d}\right)}>C\left\|U_{0}\right\|_{L^{q^{\prime}}\left(\mathbb{R}^{d}\right)} ;
$$

- (absence d'inégalités de Strichartz locales avec perte de dérivée)

$$
\begin{aligned}
\forall q \in[1,+\infty], \forall \sigma \in[0,1], & \frac{1}{2}-\frac{1}{q}>\frac{\sigma}{d} \\
& \forall T>0, \forall C>0, \exists U_{0} \in C_{0}^{\infty},\|\chi U(t)\|_{L^{1}(] 0, T\left[, L^{q}\left(\mathbb{R}^{d}\right)\right)}>C\left\|U_{0}\right\|_{D\left(P^{\sigma / 2}\right)} .
\end{aligned}
$$

Dans les inégalités précédentes, on a noté $U(t)$ la solution de l'équation (2) de condition initiale $U_{0}$ et $q^{\prime}$ l'exposant conjugué de $q$ défini par : $1 / q+1 / q^{\prime}=1$.

Remarques. - Si $d>2$, les hypothèses (617) impliquent :

$$
V \in \bigcap_{p<d / 2} L^{p}
$$

- La suite $\left(f_{n}\right)_{n \geq n_{0}}$ infirme évidemment (5) dés que $N \geq 2$.

- Il apparaîtra clairement dans la démonstration que l'on peut construire des quasi-modes d'ordre infini (c'est à dire en remplaçant $O\left(\lambda_{n}^{j-N}\right)$ par $O\left(\lambda_{n}^{-\infty}\right)$ dans (10) ) en s'autorisant une singularité un peu plus forte :

$$
\frac{|\log r|^{2+\varepsilon}}{C r^{2}} \leq V(r) \leq \frac{C|\log r|^{2+\varepsilon}}{r^{2}}, \varepsilon>0 .
$$


En s'autorisant une singularité encore plus grande, on peut imposer à la suite $\left(f_{n}\right)$ une décroissance exponentielle en $-\lambda_{n}$.

- Les inégalités de Strichartz :

$$
\|u\|_{L^{p}(] 0, T\left[, L^{q}\left(\mathbb{R}^{d}\right)\right)} \leq C\left\|u_{0}\right\|_{L^{2}\left(\mathbb{R}^{d}\right)}, p>2, \frac{2}{p}+\frac{d}{q}=\frac{d}{2},
$$

sont niées par (11), sauf le cas $q=2, p=+\infty$, trivial par conservation de la norme $L^{2}$. De même, (14) nie les inégalités de Strichartz avec perte de dérivée (cf [2]) :

$$
\|u\|_{L^{p}(] 0, T\left[L^{q}\left(\mathbb{R}^{d}\right)\right)} \leq C\left\|u_{0}\right\|_{D\left(P^{\sigma / 2}\right)}, \frac{1}{2}-\frac{1}{q}>\frac{\sigma}{d}, \sigma \in[0,1],
$$

avec des conditions supplémentaires sur $p, q$ et $\sigma$. Le cas limite $\sigma / d=1 / 2-1 / q$ de cette dernière inégalité est toujours vraie par l'inclusion de Sobolev :

$$
L^{q}\left(\mathbb{R}^{d}\right) \subset H^{\sigma}\left(\mathbb{R}^{d}\right) \subset D\left(P^{\sigma / 2}\right),
$$

et la conservation de la norme $D\left(P^{\sigma / 2}\right)$ de toute solution de (2).

Remarquons que d'après cette inclusion, (14) est encore vrai dans les espaces de Sobolev usuels, c'est à dire en remplaçant $D\left(P^{\sigma / 2}\right)$ par $H^{\sigma}\left(\mathbb{R}^{n}\right)$.

- Le potentiel $V$ et les quasi-modes $u_{n}$ étant à supports compacts, aussi proches de 0 que l'on veut, le contre-exemple précédent est également valable dans n'importe quel ouvert borné, pour un Laplacien de Neumann ou de Dirichlet.

- Des exemples d'équations de Schrödinger ne vérifiant pas de dispersion ou d'inégalités de Strichartz sont également construits dans [1] et [4. Dans les deux cas, le problème vient de la métrique définissant le Laplacien. Mentionnons aussi que l'idée d'utiliser des quasi-modes en relation avec des inégalités de Strichartz est due à M. Zworski (voir [8] ).

Précisons encore que pour le potentiel $V$ du théorème, on peut définir $P$ sans ambiguité. L'opérateur $-\Delta+V$ a un sens sur $C_{0}^{\infty}\left(\mathbb{R}^{d} \backslash\{0\}\right)$, et est essentiellement auto-adjoint sur cet espace (cf par exemple le théorème de Kalf, Walter, Schmincke et Simon cité dans [7] page 186). On appelle $P$ son unique extension auto-adjointe, qui est bien sûr positive.

L'auteur tient à remercier Nicolas Burq, son directeur de recherche, et Clotilde Fermanian Kammerer pour leur aide précieuse.

Le reste du texte est consacré aux démonstrations des résultats présentés dans cette introduction.

\section{DÉmonstration du COROLlaire.}

D'après l'inégalité de Hölder, si $v$ est une fonction sur $\mathbb{R}^{d}$ avec un support à volume fini $B$,

$$
\|v\|_{L^{q_{0}}} \leq B^{1 / q_{0}-1 / q}\|v\|_{L^{q}}, \quad q>q_{0} .
$$

Posons $U_{n}(t)=e^{-i \lambda_{n}^{2} t} u_{n}$. Alors :

$$
\begin{gathered}
i \partial_{t} U_{n}-P U_{n}=-e^{-i \lambda_{n}^{2} t} f_{n}, \quad U_{n\lceil t=0}=u_{n} \\
U_{n}(t)=e^{-i t P} u_{n}+i e^{-i \lambda_{n}^{2} t} \int_{0}^{t} e^{i(s-t) P} f_{n} d s .
\end{gathered}
$$

Supposons que (11) est fausse. D'après (16):

$$
\int_{0}^{T}\left\|\chi U_{n}(t)\right\|_{L^{q}\left(\mathbb{R}^{d}\right)} d t \leq \int_{0}^{T}\left\|\chi e^{-i t P} u_{n}\right\|_{L^{q}\left(\mathbb{R}^{d}\right)} d t+\int_{0}^{T} \int_{0}^{t}\left\|\chi e^{i(s-t) P} f_{n}\right\|_{L^{q}\left(\mathbb{R}^{d}\right)} d s d t .
$$

Puisque le support de $u_{n}$ se concentre en 0 , et que $\chi(0) \neq 0$, le terme de gauche de cette inégalité est minoré pour $n$ assez grand par :

$$
\int_{0}^{T}\left\|u_{n}\right\|_{L^{q}\left(\mathbb{R}^{d}\right)} d t \geq \frac{1}{C}\left(\frac{\log \lambda_{n}}{\lambda_{n}}\right)^{d\left(1 / q-1 / q_{0}\right)}\left\|u_{n}\right\|_{L^{q_{0}\left(\mathbb{R}^{d}\right)}} .
$$


Quant au terme de droite, il est dominé d'après la négation de (11) par :

$$
\left\|u_{n}\right\|_{L^{q_{0}\left(\mathbb{R}^{d}\right)}}+\left\|f_{n}\right\|_{L^{q_{0}\left(\mathbb{R}^{d}\right)}} .
$$

On en déduit, en utilisant (10) :

$$
\left(\frac{\lambda_{n}}{\log \lambda_{n}}\right)^{d\left(1 / q_{0}-1 / q\right)}\left\|u_{n}\right\|_{L^{q_{0}}}=O\left(\left\|u_{n}\right\|_{L^{q_{0}}}+\lambda_{n}^{-N}\right)
$$

ce qui donne la contradiction annoncée dés que $N \geq 1$ et $q_{0}<q$, car d'après (9), la norme de $u_{n}$ dans $L^{q_{0}}$ est minorée par 1 .

Par les inégalités de Sobolev, (11) implique (12) en prenant, dans (11), $q_{0}=2$ et $q>2$ assez proche de 2. Donc (12) est vraie aussi.

Supposons maintenant la négation de (13). Alors, par (16) :

$$
\begin{aligned}
\left\|\chi u_{n}(T)\right\|_{L^{q}\left(\mathbb{R}^{d}\right)} & \leq C\left\|u_{n}\right\|_{L^{q^{\prime}\left(\mathbb{R}^{d}\right)}}+\int_{0}^{T}\left\|\chi e^{i(s-T)} f_{n}\right\|_{L^{1}\left(\mathbb{R}^{d}\right)} d s \\
& \leq C\left\|u_{n}\right\|_{L^{q^{\prime}\left(\mathbb{R}^{d}\right)}}+C\left\|f_{n}\right\|_{L^{2}\left(\mathbb{R}^{d}\right)},
\end{aligned}
$$

où on a simplement majoré, pour obtenir la deuxième inégalité, la norme $L^{q^{\prime}}$ sur le support de $\chi$ par la norme $L^{2}$, à une constante multiplicative près. On obtient à nouveau une contradiction par (15) et en utilisant $q^{\prime}<q$.

Il reste à démontrer (14). Dans le cas contraire, on aurait, par (16) :

$$
\int_{0}^{T}\left\|u_{n}(t)\right\|_{L^{q}\left(\mathbb{R}^{d}\right)} d t \leq C\left\|u_{n}\right\|_{D\left(P^{\sigma / 2}\right)}+\int_{0}^{T} \int_{0}^{t}\left\|e^{i(s-t) P} f_{n}\right\|_{D\left(P^{\sigma / 2}\right)} d s d t .
$$

Bien sûr, $e^{i(s-t) P}$ envoie isométriquement le domaine de $P^{\sigma / 2}$ sur lui-même. On a :

$$
\left\|f_{n}\right\|_{D\left(P^{1 / 2}\right)}^{2}=\int\left|\nabla f_{n}(x)\right|^{2} d x+\int(1+V(x))\left|f_{n}(x)\right|^{2} d x .
$$

Sur le support de $u_{n}$, donc sur celui de $f_{n},|x| \geq \frac{\log \lambda_{n}}{C \lambda_{n}}$. En utilisant la majoration de $V$ donnée par (17), on obtient que sur le support de $f_{n}$ :

$$
|V(r)| \leq C \frac{\lambda_{n}^{2}}{\left(\log \lambda_{n}\right)^{2}}\left(\log \log \lambda_{n}-\log \lambda_{n}\right)^{2} \leq C \lambda_{n}^{2}
$$

On en déduit, en utilisant (10) avec $N \geq 2$ :

$$
\left\|f_{n}\right\|_{D\left(P^{1 / 2}\right)} \leq C\left(\left\|\nabla f_{n}\right\|_{L^{2}}+\lambda_{n}\left\|f_{n}\right\|_{L^{2}}\right) \leq C \lambda_{n}^{-1} \text {. }
$$

De plus, l'équation $P u_{n}-\lambda_{n}^{2} u_{n}=f_{n}$ implique, en utilisant à nouveau les propriétés (9) et (10) des normes de $u_{n}$ et $f_{n}$ :

$$
\begin{aligned}
\left\|u_{n}\right\|_{D\left(P^{1 / 2}\right)}^{2} & \leq\left\|f_{n}\right\|_{L^{2}}\left\|u_{n}\right\|_{L^{2}}+\lambda_{n}^{2}\left\|u_{n}\right\|_{L^{2}}^{2} \\
& \leq C \lambda_{n}^{2}\left\|u_{n}\right\|_{L^{2}}^{2} .
\end{aligned}
$$

Par interpolation sur les normes, on obtient, puisque $\sigma$ est compris entre 0 et 1 :

$$
\left\|u_{n}\right\|_{D\left(P^{\sigma / 2}\right)} \leq C \lambda_{n}^{\sigma}\left\|u_{n}\right\|_{L^{2}}
$$

D'après (18), (19) et l'inégalité (17) :

$$
\left\|u_{n}\right\|_{L^{q}} \leq C \lambda_{n}^{\sigma}\left\|u_{n}\right\|_{L^{2}}+o(1) .
$$

Donc avec (15) :

$$
\left(\frac{\lambda_{n}}{\log \lambda_{n}}\right)^{d / 2-d / q}\left\|u_{n}\right\|_{L^{2}} \leq C \lambda_{n}^{\sigma}\left\|u_{n}\right\|_{L^{2}}+o(1)
$$

inégalité absurde car $\frac{d}{2}-\frac{d}{q}>\sigma$. 


\section{DÉmonstration DU THÉORÈme.}

Notons $r=|x|$ et ' la dérivée $\frac{d}{d r}$. On cherche $V, \lambda_{n}, u_{n}$ et $f_{n}$, radiales tels que :

$$
f_{n}(r)=-u_{n}^{\prime \prime}(r)-\frac{d-1}{r} u_{n}^{\prime}(r)+V(r) u_{n}(r)-\lambda_{n}^{2} u_{n}(r),
$$

avec $f_{n}$ petit à l'infini. On commence par changer de fonctions pour éliminer la dérivée première dans cette expression. En posant :

$$
u_{n}=r^{-\frac{d-1}{2}} v_{n}, \quad f_{n}=r^{-\frac{d-1}{2}} g_{n}, \quad W=V+\frac{d^{2}-4 d+3}{4 r^{2}} .
$$

L'équation (20) devient :

$$
g_{n}(r)=-v_{n}^{\prime \prime}+W v_{n}-\lambda_{n}^{2} v_{n}
$$

Soient :

$$
y(s)=e^{-\sqrt{s^{2}+1}}, b(s)=-\frac{1}{\left(s^{2}+1\right)^{3 / 2}}+\frac{s^{2}}{s^{2}+1},
$$

solutions $C^{\infty}$ sur $\mathbb{R}$ de :

$$
\begin{gathered}
-y^{\prime \prime}(s)+b(s) y(s)=0 \\
y(s)>0, \forall j \in \mathbb{N}, \quad\left|y^{(j)}(s)\right| \leq C_{j} e^{-|s|} \\
|b(s)| \leq 1 .
\end{gathered}
$$

On notera, pour 2 paramètres réels $\omega$ et $a$ :

$$
y_{\omega, a}(r)=y(\omega(r-a)), \quad b_{\omega, a}(r)=\omega^{2} b(\omega(r-a)),
$$

solutions de :

$$
-y_{\omega, a}^{\prime \prime}+b_{\omega, a} y_{\omega, a}=0
$$

Soit $q(\lambda)>0$ définie au voisinage de $+\infty$, strictement croissante et vérifiant :

$$
\begin{aligned}
& \lim _{\lambda \rightarrow+\infty} q(\lambda)=+\infty \\
& \lim _{\lambda \rightarrow+\infty} \frac{q(\lambda)}{\lambda}=0 .
\end{aligned}
$$

On définit la suite $\left(\lambda_{n}\right)_{n \geq n_{0}}$, qui tend vers $+\infty$ plus vite que $10^{n}$ par :

$$
10^{n}=q\left(\lambda_{n}\right) .
$$

Soit une troncature : $\chi \in C_{0}^{\infty}(]-1,1[), \chi=1$ sur $\left[-\frac{1}{2}, \frac{1}{2}\right]$.

On notera :

$$
\begin{gathered}
\psi_{n}(r)=\chi\left(10^{n} r\right)-\chi\left(10^{n+1} r\right), \\
\chi_{n}(r)=\chi\left(10^{n+1}\left(r-\frac{3}{10^{n+1}}\right)\right),
\end{gathered}
$$




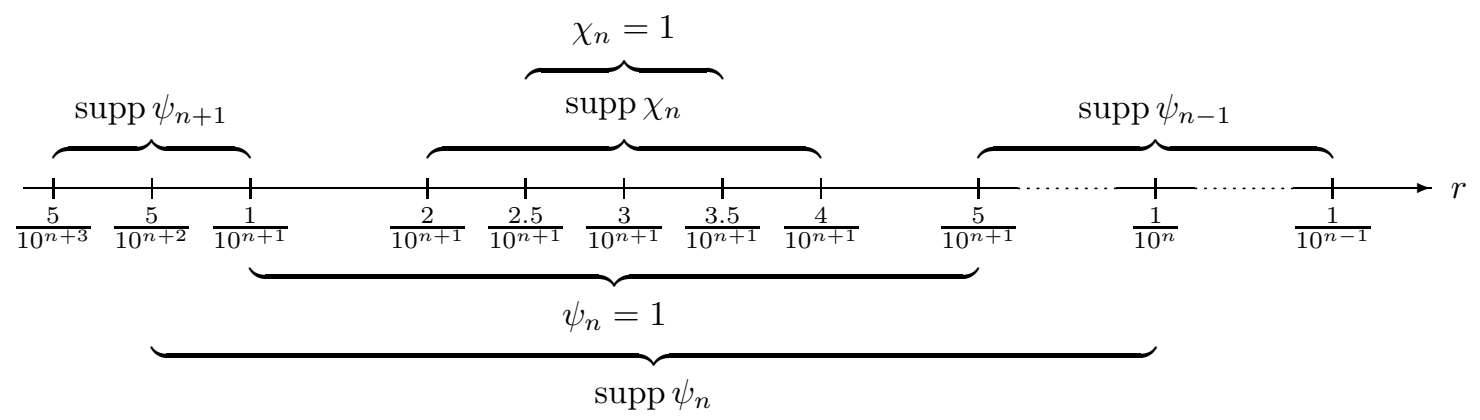

FIG. 1 - Troncatures autour de $r=3 / 10^{n}+1$

Les $\psi_{n}$ forment une partition de l'unité au voisinage de 0 , et chacun des $\chi_{n}$ localise sur le support de $\psi_{n}$, en dehors du support des autres $\psi_{j}, j \neq n$ :

$$
\begin{gathered}
\operatorname{supp} \psi_{n} \subset\left[\frac{5}{10^{n+2}}, \frac{1}{10^{n}}\right] \\
\frac{1}{10^{n+1}} \leq r \leq \frac{5}{10^{n+1}} \Rightarrow \psi_{n}(r)=1 \\
\sum_{n \geq n_{0}} \psi_{n}(r)=\chi\left(10^{n_{0}} r\right) \\
\operatorname{supp} \chi_{n} \subset\left[\frac{2}{10^{n+1}}, \frac{4}{10^{n+1}}\right] \subset\left\{\psi_{n}=1\right\} \\
\frac{25}{10^{n+2}} \leq r \leq \frac{35}{10^{n+2}} \Rightarrow \chi_{n}(r)=1 .
\end{gathered}
$$

On notera $y_{n}$ et $b_{n}$ les fonctions :

$$
\begin{gathered}
y_{n}(r)=y_{\frac{\lambda_{n}}{2}, \frac{3}{10^{n+1}}}(r)=y\left(\frac{\lambda_{n}}{2}\left(r-\frac{3}{10^{n+1}}\right)\right) \\
b_{n}(r)=b_{\frac{\lambda_{n}}{2}, \frac{3}{10^{n+1}}}(r)=\frac{\lambda_{n}^{2}}{4} b\left(\frac{\lambda_{n}}{2}\left(r-\frac{3}{10^{n+1}}\right)\right) .
\end{gathered}
$$

On peut voir chacun des $-b_{n}$ comme un puits de potentiel qui, par l'équation (221), concentre l'énergie de $y_{n}$ autour du point $3 / 10^{n+1}$. On va construire $W$ pour que l'équation $g_{n}(r)=0$ s'écrive, sur un petit intervalle autour de $3 / 10^{n+1}$ (qui inclura le support de $v_{n}$ ) $-v_{n}^{\prime \prime}+b_{n} v_{n}=0$. La taille de cette intervalle sera de l'ordre de $10^{-n}$, tendant moins vite vers 0 que le paramètre $\omega=\lambda_{n} / 2$ de l'équation. En définissant $v_{n}$ comme une troncature de $y_{n}$, la décroissance exponentielle de $y$ à l'infini induira grâce à la différence d'ordre de ces deux échelles, des termes d'erreur assez petits pour impliquer (9) et (10).

Posons (cf figure) :

$$
\begin{gathered}
W(r)=\sum_{n \geq n_{0}} \psi_{n}(r)\left(b_{n}(r)+\lambda_{n}^{2}\right) \\
v_{n}(r)=\alpha_{n} y_{n}(r) \chi_{n}(r),
\end{gathered}
$$

où les $\alpha_{n}$ sont des constantes strictement positives à choisir. Les fonctions $g_{n}, u_{n}$ et $f_{n}$, ainsi que le potentiel $V$ sont alors définies par (2011) et (21). 


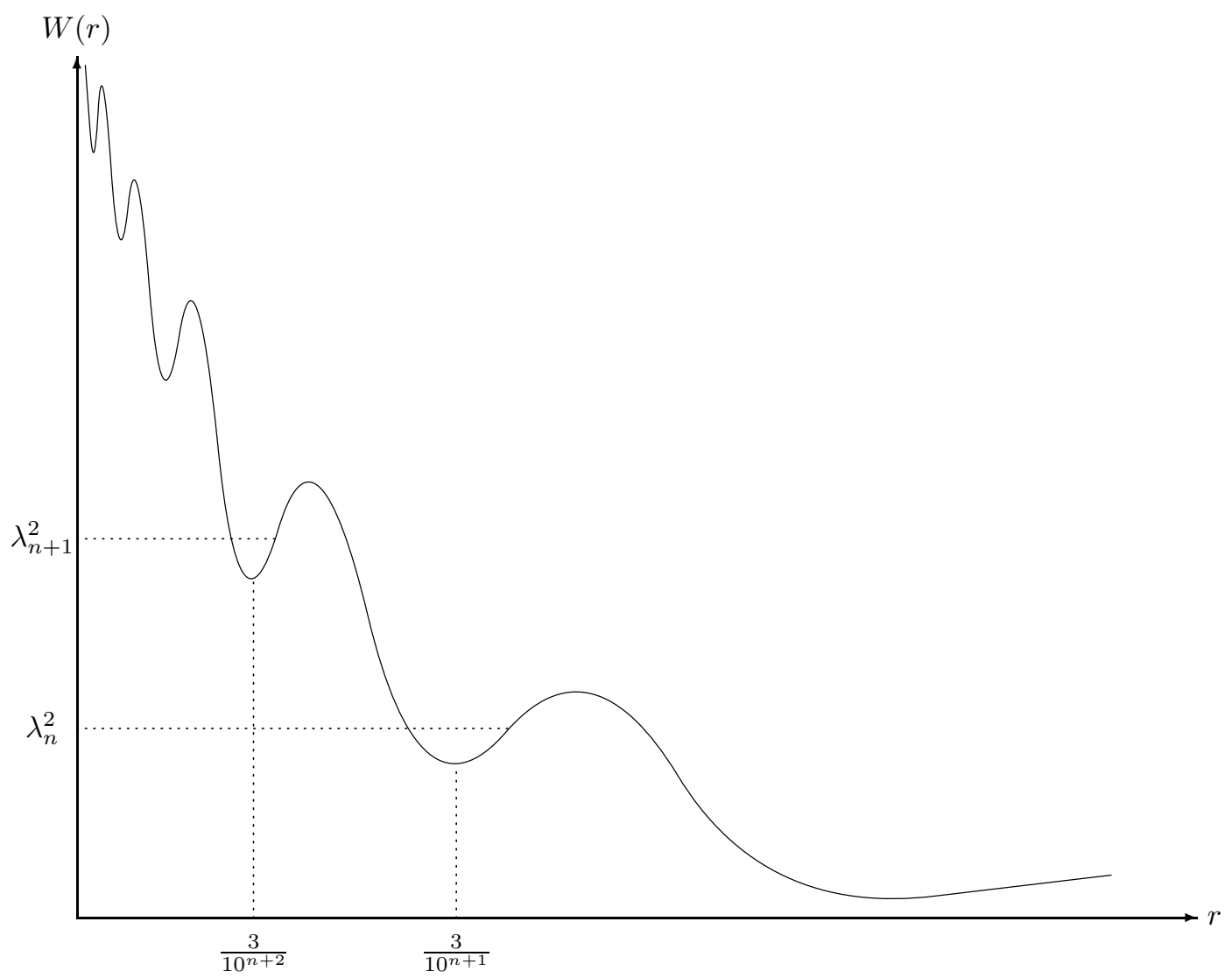

FIG. 2 - Le potentiel W

Lemme 1. On a les inégalités suivantes:

$$
\begin{gathered}
\forall j \in \mathbb{N}, \exists C>0,\left\|\frac{d^{j} f_{n}}{d r^{j}}\right\|_{L^{\infty}\left(\mathbb{R}^{d}\right)} \leq C \alpha_{n} \lambda_{n}^{1+j}\left(q\left(\lambda_{n}\right)\right)^{\frac{d+1}{2}} e^{-\frac{\lambda_{n}}{20 q\left(\lambda_{n}\right)}} \\
\left\|u_{n}\right\|_{L^{1}\left(\mathbb{R}^{d}\right)} \geq \frac{\alpha_{n}}{C} \lambda_{n}^{-1}\left(q\left(\lambda_{n}\right)\right)^{-\frac{d-1}{2}} .
\end{gathered}
$$

Démonstration. D'après (28), sur le support de $\chi_{n}, W$ vaut exactement $b_{n}+\lambda_{n}^{2}$. On en déduit :

$$
g_{n}(r)=-v_{n}^{\prime \prime}(r)+b_{n}(r) v_{n}(r) .
$$

Soit, en utilisant l'équation (221) :

$$
f_{n}(r)=r^{-\frac{d-1}{2}} g_{n}(r)=r^{-\frac{d-1}{2}} \alpha_{n}\left(y_{n}(r) \chi_{n}^{\prime \prime}(r)+2 y_{n}^{\prime}(r) \chi_{n}^{\prime}(r)\right) .
$$

La dérivée $j$-ème de $f_{n}$ est donc de la forme :

$$
\frac{d^{j} f_{n}}{d r^{j}}=\sum_{j_{1}+j_{2}+j_{3}=j+1} \beta_{j_{1}, j_{2}, j_{3}} \frac{d^{j_{1}}}{d r^{j_{1}}} y_{n} \frac{d^{j_{2}}}{d r^{j_{2}}} \chi_{n}^{\prime} \frac{d^{j_{3}}}{d r^{j_{3}}} r^{-\frac{d-1}{2}} .
$$

Sur le support de $\chi_{n}^{\prime}$, on a :

$$
\left|r-\frac{3}{10^{n+1}}\right|>\frac{1}{10^{n+1}}=\frac{1}{10 q\left(\lambda_{n}\right)} .
$$


Donc d'après les majorations (23) :

$$
\begin{aligned}
\left|y_{n}^{\left(j_{1}\right)}(r)\right| & \leq C \lambda_{n}^{\left(j_{1}\right)} e^{-\frac{\lambda_{n}}{2}\left|r-\frac{3}{10^{n}+1}\right|} \\
& \leq \lambda_{n}^{j_{1}} e^{-\frac{\lambda_{n}}{20 q\left(\lambda_{n}\right)}}
\end{aligned}
$$

De plus :

$$
\begin{gathered}
\left|\chi_{n}^{\left(j_{2}+1\right)}\right| \leq C\left(q\left(\lambda_{n}\right)\right)^{j_{2}+1} \leq C \lambda_{n}^{j_{2}} q\left(\lambda_{n}\right) \\
\left|\left(\frac{d}{d r}\right)^{j_{3}}\left(r^{-\frac{d-1}{2}}\right)\right| \leq C\left(q\left(\lambda_{n}\right)\right)^{\frac{d-1}{2}+j_{3}} \leq C \lambda_{n}^{j_{3}}\left(q\left(\lambda_{n}\right)\right)^{\frac{d-1}{2}},
\end{gathered}
$$

car sur le support de $\chi_{n}, r \geq \frac{1}{10^{n+1}}$. En majorant la somme (37) par ces trois dernières inégalités, on obtient exactement (34). (37), on obtient exactement (34).

Démontrons (35). Par définition de $y_{n}$ :

$$
\frac{\lambda_{n}}{2}\left|r-\frac{3}{10^{n}}\right| \leq \frac{1}{2} \Rightarrow y_{n}(r) \geq m=\sup _{|s| \leq \frac{1}{2}}|y(s)| .
$$

De plus, si cette dernière condition sur $r$ est vérifiée, et si $n$ est assez grand on a $\chi_{n}(r)=1$ et donc :

$$
u_{n}(r)=r^{-\frac{d-1}{2}} v_{n}(r)=r^{-\frac{d-1}{2}} \alpha_{n} y_{n}(r)
$$

D'où :

$$
\begin{aligned}
\left\|u_{n}\right\|_{L^{1}} & \geq m \alpha_{n} \int_{\lambda_{n}\left|r-\frac{3}{10^{n}}\right| \leq 1} r^{-\frac{d-1}{2}} r^{d-1} d r \\
& \geq \frac{\alpha_{n}}{C}\left(10^{-n}\right)^{\frac{d-1}{2}} \lambda_{n}^{-1} .
\end{aligned}
$$

Choisissons un $M>1$ et posons :

$$
q(\lambda)=\frac{\lambda}{M \log \lambda} .
$$

La fonction $q$, positive et strictement croissante au voisinage de $+\infty$, vérifie bien les hypothèses (25) et (26). De plus, d'après le lemme 1 et en majorant grossièrement $q\left(\lambda_{n}\right)$ par $\lambda_{n}$ :

$$
\begin{gathered}
\left\|\frac{d^{j} f_{n}}{d r^{j}}\right\|_{L^{\infty}} \leq C \alpha_{n} \lambda_{n}^{\frac{d+3}{2}+j-\frac{M}{20}} \\
\left\|u_{n}\right\|_{L^{1}} \geq \frac{\alpha_{n}}{C} \lambda^{\frac{d+1}{2}}
\end{gathered}
$$

avec des nouvelles constantes $C$ qui dépendent éventuellement de $M$. Les conditions (92) et (10) du théorème sont donc respectées avec un bon choix des constantes $M$ et $\alpha_{n}$. Le support de $u_{n}$ est confondu avec celui de $\chi_{n}$, qui est effectivement de la forme :

$$
\left\{c_{1} \frac{\log \lambda_{n}}{\lambda_{n}} \leq r \leq c_{2} \frac{\log \lambda_{n}}{\lambda_{n}}\right\},
$$

si $\chi$ est bien choisie. Il reste à vérifier l'assertion (7) sur le potentiel. On commence par évaluer la fonction réciproque de $q$.

Lemme 2. Soit q est définie par (38). Alors:

$$
q(\lambda) \log (q(\lambda)) \sim \frac{\lambda}{M}, \lambda \rightarrow+\infty .
$$




\section{Démonstration.}

$$
\begin{aligned}
q(\lambda) \log q(\lambda) & =q(\lambda) \log \left(\frac{\lambda}{M \log \lambda}\right) \\
& =q(\lambda)(\log \lambda-\log \log \lambda-\log M) \\
& \sim q(\lambda) \log \lambda,
\end{aligned}
$$

quand $\lambda$ tend vers $+\infty$.

Sur le support de $\psi_{n}$, puisque $q\left(\lambda_{n}\right)=10^{n}$,

$$
\begin{gathered}
\frac{1}{20 r} \leq q\left(\lambda_{n}\right) \leq \frac{1}{r} \\
\frac{1}{20 r}|\log r-\log 20| \leq q\left(\lambda_{n}\right) \log \left(q\left(\lambda_{n}\right)\right) \leq \frac{1}{r}|\log r|,
\end{gathered}
$$

(l'application $s \mapsto s \log s$ étant croissante pour $s>e^{-1}$ ). Soit, en utilisant le lemme 2 et en se plaçant assez près de 0 :

$$
C^{-1} \frac{1}{r}|\log r| \leq \lambda_{n} \leq C \frac{1}{r}|\log r| .
$$

Donc, par la définition (32) de $W(r)$, et comme $\left|b_{n}\right|$ est majoré par $\lambda_{n}^{2} / 4$ :

$$
C^{-1} \frac{|\log r|^{2}}{r^{2}} \sum_{n \geq n_{0}} \psi_{n}(r) \leq W(r) \leq C \frac{|\log r|^{2}}{r^{2}} \sum_{n \geq n_{0}} \psi_{n}(r),
$$

ce qui donne l'inégalité (7) sur le potentiel $W$ compte tenu de (27). On en déduit évidemment (7) sur $V$.

Remarque. Pour avoir des quasi-modes d'ordre infini il suffisait de prendre $q(\lambda)=\frac{\lambda}{(\log \lambda)^{1+\varepsilon}}$, et de modifier le lemme 2 en conséquence.

\section{RÉFÉRENCES}

[1] V. Banica. Dispersion and Strichartz inequalities for Schrödinger equations with singular coefficients. A paraître au SIAM Journal of Mathematical Analysis.

[2] N. Burq, P. Gérard, N. Tzvetkov. On nonlinear Schrödinger equations in exterior domains. Preprint.

[3] N. Burq, F. Planchon, J.G. Stalker, A.Shadi Tahvildar-Zadel. Strichartz estimates for the wave and Schrödinger equations with the inverse-square potential. Preprint.

[4] C. Castro, E. Zuazua. Concentration and lack of observability of waves in highly heterogeneous media. Arch. Ration. Mech. Anal. 164 (2002), No 1, 39-72.

[5] T. Duyckaerts. Inégalités de résolvante pour des potentiels multipolaires. Travail en cours.

[6] A. Ruiz, L. Vega. Local regularity of solutions to wave with time-dependent potentials. Duke Math. J. 76 (1994) No 3 p. 913-940.

[7] M. Reed, B. Simon. Methods of modern mathematical physics. 2, Fourier analysis, self-adjointness. San Diego CA London Sydney : Academic Press, 1975.

[8] H. F. Smith, C.D. Sogge, Global Strichartz estimates for nontrapping perturbations of the Laplacian. Comm. Partial Differential Equations 25 (2000), No 11-12, 2171-2183. Arch. Ration. Mech. Anal. 164 (2002), No 1, 39-72.

Université de Paris-Sud, BÂtiment 430, 91405 Orsay Cedex

E-mail address: Thomas.Duyckaerts@math.u-psud.fr 